\title{
Gastric antral vascular ectasia (GAVE) and hereditary hemorrhagic telangiectasia (HHT): two different conditions, one treatment
}

\author{
Sara Santos $^{1}$ (D) $\cdot$ Carlos Bernardes $^{1} \cdot$ Verónica Borges $^{1} \cdot$ Gonçalo Ramos $^{1}$ \\ Received: 21 August 2019 / Accepted: 12 November 2019 / Published online: 26 November 2019 \\ (C) Springer-Verlag GmbH Germany, part of Springer Nature 2019
}

Dear Editor,

Vascular lesions of the digestive tract are among the most frequent causes of gastrointestinal bleeding and irondeficiency anemia. We write concerning a particular entitygastric antral vascular ectasia (GAVE) - which is endoscopically characterized by parallel red stripes, angiomatous lesions at the antral mucosal folds, frequently referred to as watermelon stomach. Besides this typical location, it may rarely be found in the cardia, duodenum, jejunum, and rectum. The pathogenesis of this entity is still obscure. However, it is associated with certain comorbidities, such as autoimmune disease, liver cirrhosis, or chronic renal failure [1, 2].

In patients with active hemorrhage or anemia due to chronic bleeding from GAVE, the available evidence favors endoscopic therapy with argon plasma coagulation (APC) or Nd:YAG. Several medical strategies to reduce GI bleeding have been described, namely hormonal therapy, octreotide, tranexamic acid, thalidomide, or prednisolone, none of them showing ideal outcomes due to severe side effects or conflicting/scarce results [1, 3-6].

We report the case of a 49-year-old male undergoing dialysis due to membranoproliferative glomerulonephritis. For the previous 2 years, the patient reported frequent episodes of melena and anemia, with recurrent need for blood transfusion. He was diagnosed with gastric antral vascular angiectasia (GAVE) and submitted to three gastric argon plasma treatments and a trial of thalidomide, which was withdrawn due to a pulmonary embolism.

Sara Santos

saramendezleiria@hotmail.com

1 Centro Hospitalar de Lisboa Central, Lisbon, Portugal
He presented to our emergency department with complaints of fatigue and melena for the previous 2 weeks. Laboratory data revealed low hemoglobin value $(6.3 \mathrm{~g} / \mathrm{dL})$ with low median corpuscular volume $(65 \mathrm{fL})$. The patient was submitted to upper endoscopy, showing multiple gastric angiodysplasias arranged in radiating streaks (Fig. 1) with active bleeding, and argon plasma coagulation was performed. Nevertheless, during hospital stay (1 month), he continued to require frequent transfusion (10 $\mathrm{U}$ of packed red blood cells/ month) despite undergoing three other therapeutic endoscopies. Further workup, namely a capsule endoscopy, revealed countless angiectasias in the jejunum, with active hemorrhage.

Facing this severe and refractory gastrointestinal bleeding, in which endoscopic therapy was insufficient, whether due to the limited depth of coagulation of APC or the extensive involvement of the small bowel not usually seen in GAVE patients, therapy with bevacizumab was proposed. Although there were no published cases with this drug in GAVE, our experience and the available evidence of this drug in patients with hereditary-hemorrhagic-telangiectasia (HHT) were supportive and this patient needed an early clinical effect, which is usually obtained from this drug. Based on the pre-existing data and the elimination half-life-18 to 20 days - we started the regimen commonly used in HHT, $5 \mathrm{mg} / \mathrm{kg}$ every 3 weeks. Previously published data also demonstrated similar pharmacokinetic parameters between hemodialyzed and normal renal function patients $[7,8]$.

After the first administration, a single transfusion was necessary 2 weeks later, and since then a gradual rise in the hemoglobin values was reported (Graphic 1) as well as normalization of serum iron (ferritin $97 \mathrm{ng} / \mathrm{mL}$; transferrin saturation of $22 \%$ ). Parallel improvement in general status and tolerance for daily activities was stated and after 1 year of therapy the patient remains free from blood replacement therapy, without 
Fig. 1 Upper digestive endoscopy revealing angiodysplasias arranged in radiating streaks in the antrum

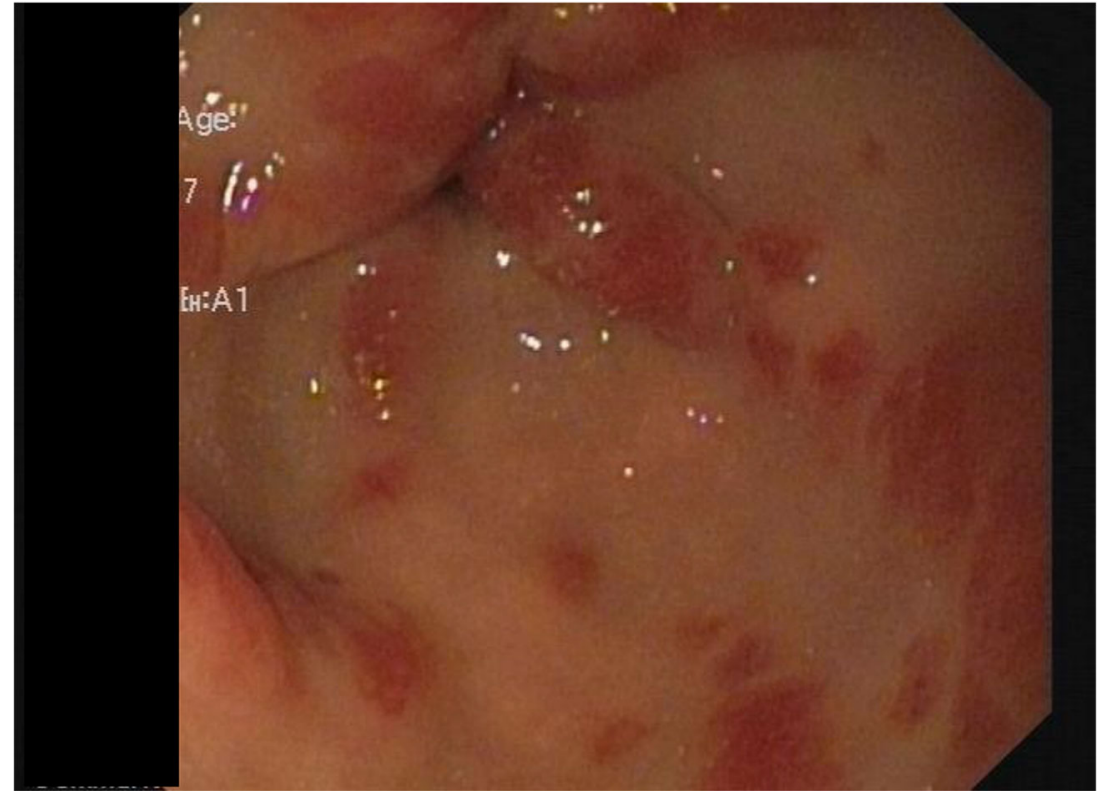

further episodes of melena or hospital admissions for this condition; in addition, there were no adverse events to report.

Although the etiology of GAVE is unclear, the results observed with bevacizumab suggest a role for VEGF, similarly to what occurs in other vascular abnormalities; however, further studies are required. Most of the treatment strategies currently considered in GAVE are extrapolations of those being used in other gastrointestinal vascular diseases. While bevacizumab has been proved to be effective in other diseases, as Rendu-Osler-Weber, its use in GAVE has not been explored, being yet to define the optimum dose/interval, as well as the duration of the treatment after remission.

In refractory cases, with extensive bowel involvement, bevacizumab may be considered, having showed in this patient an almost immediate, significant, and sustained response.

Graphic 1 Hemoglobin levels before and after bevacizumab 


\section{Compliance with ethical standards}

Conflict of interest The authors declare that they have no conflict of interest.

Ethical approval All procedures performed in studies involving human participants were in accordance with the ethical standards of the institutional and/or national research committee and with the 1964 Helsinki declaration and its later amendments or comparable ethical standards.

Informed consent Informed consent was obtained from all individual participants included in the study.

\section{References}

1. Hsu WH, Wang YK, Hsieh MS, et al.: Insights into the management of gastric antral vascular ectasia (watermelon stomach), Therap Adv Gastroenterol. 2018; 14;11:1756283X17747471

2. Alkhormi AM, Memon MY, Alqarawi A (2018) Gastric antral vascular ectasia: a case report and literature review. J Transl Int Med $6(1): 47-51$
3. Sebastian S, O'Morain CA, Buckley MJ (2003) Review article: current therapeutic options for gastric antral vascular ectasia. Aliment Pharmacol Ther 18:157-165

4. Swanson E, Mahgoub A, MacDonald R, Shaukat A (2014) Medical and endoscopic therapies for angiodysplasia and gastric antral vascular ectasia: a systematic review. Clin Gastroenterol Hepatol 12(4): 571-582

5. Barbara G1 De Giorgio R, Salvioli B: Unsuccessful octreotide treatment of the watermelon stomach. J Clin Gastroenterol. 1998; 26(4): $345-6$

6. Nardone G, Rocco A, Balzano T, Budillon G (1999) The efficacy of octreotide therapy in chronic bleeding due to vascular abnormalities of the gastrointestinal tract. Aliment Pharmacol Ther 13(11):14291436

7. Garnier-Viougeat N, Rixe O, Paintaud G (2007 Mar) Pharmacokinetics of bevacizumab in haemodialysis. Nephrol Dial Transplant 22(3):975

8. Epperla N, Hocking W (2015) Blessing for the bleeder: bevacizumab in hereditary hemorrhagic telangiectasia. Clin Med Res 13(1):32-35

Publisher's note Springer Nature remains neutral with regard to jurisdictional claims in published maps and institutional affiliations. 